Presented at the Third National Conference on Synchrotron Radiation Instrumentation at Brookhaven National Laboratory, Sept. 12-14, 1983.

USE OF SEMICONDUCTOR DETECTORS WITH SYNCHROTRON RADIATION

A. C. Thompson, F. S. Goulding and J. T. Walton Lawrence Berkeley Laboratory University of California Berkeley, CA 94720

Sep tember 1983

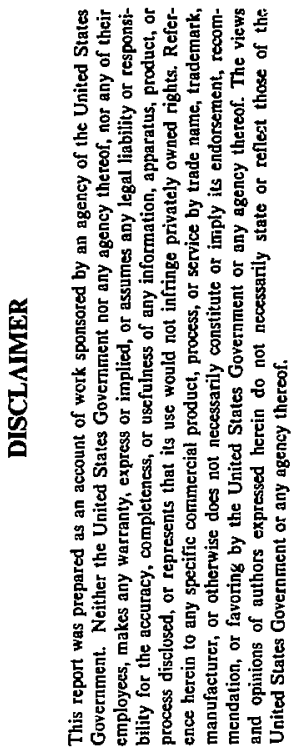

This work was supported by the Director's Office of Energy Research, Office of Health and Environmental Research, U.S. Department of Energy under Contract No. DE-ACO3-76SF00098. 


\title{
USE OF SEMICONDUCTOR DETECTORS WITH SYNCHROTRON RADIATION
}

\author{
AC. Thompeon, F.S. Goulding and J.T. Walton \\ Lawrence Berkeley Laboratory \\ University of California \\ berkeley, Ca 94720.
}

\begin{abstract}
Silicon and germanium semiconductor detectors are widely used as photon and charged-particle spectrometers. Semiconductor detectors combine wide energy bandwidth with good energy resolution to make useful spectrometers for use with the high-intensity $x$-ray sources available at synchrotron facilities. High-quality silicon and germanium single-crystal starting material is now available for the fabrication of large-volume detectors. Techniques have also been developed to reliabiy make detectors with passivated surfaces, thin windows and complex geometries. New low-noise preaniplifiers and pulse-shaping networks allow higher counting rates and improved fulse throughput. For applications where accurate flux measurements of intense photon beams are required, a semiconductor detector with a current-measuring voltageto-frequency converter provides a simple system which has excellent linearity and stability. Examples are given where semiconductor detectors have been used in experiments at synchrotron facilities.
\end{abstract}




\section{INTRODUCTION}

Silicon and germanium detectors are widely used as detectors of photons and charged particles. The wide energy bandwidth of these detectors allows the simultaneous measureinent of many different photon energies. These spectrometers cover the photon energy range from $200 \mathrm{eV}$ to several $\mathrm{MeV}$. In this paper only the use of these detectors for photon detection up to $100 \mathrm{keV}$ will be presented since this is the normal range of synchrotron x-ray sources.

Increasing availabjlity of larger and higher-quality silicon single crystals is leading to wider applications for silicon detectors. In addition, many of the processing techniques which have been developed primarily for integrated circuit manufacturing are now used in detector fabrication.

Silicon detectors less than $0.5 \mathrm{~mm}$ are generally used only as charged partisie detectors while silicon detectors 0.5 to $6 \mathrm{~mm}$ thick are used as both charged-particle and low-energy (<SO keV) photon detectors. For charged particles they are operated at room temperature. For low-energy photons they are cooled to reduce the thermal leakage current and used with cooled low-noise preampliers.

High-purity germanium detectors have totally replated lithium-drifted detectors, since reliable techniques have been developed for purifying germanium. Germanium detectors are usually made from high-purity single crystals which are prepared especially for radiation detectors. These single crystals are made by first refining the starting material using a multi-pass RF float-zone process. A single crystal is then g-own using the Czochralski method. The concentration of elecirically active impurities of the useful part of these crystals is generally irt the region of $10^{10} \mathrm{~cm}^{3}$.

Germanium detectors are used primarily as high energy photon detectors. They are generally not used below $20 \mathrm{keV}$ because of the interference from peaks due to escape of the germanium $\mathrm{K}$ flucrescence photon. This effect is illustrated in Figure 1 in which the efficiency of both a $3 \mathbf{m m}$ thick silicon detector and a $5 \mathrm{~mm}$ germanium detector are shown. Also shown in this figure is the low energy efficiency with a thin silicon dead layer, with a $25 \mu \mathrm{m}$ Be window and with $2 \mathrm{~cm}$ of air between the source and the detecter. Germanium detectors must be cooled to around $77 \mathrm{deg} \mathrm{K}$ to reduce leakage current and they of ten have a cooled preamplifier to give improved energy resolution. For use below $100 \mathrm{keV}$ a planar detector geometry is used while for higher energy photon detectors a coaxial geometry is often used to give large volume detectors.

The development of cooled, transistor reset FET preamplifiers and new pulse shaping networks has allowed higher counting rates with good energy resolution and better pulse throughput with improved piliup rejection $[1,2]$. For applications where accurate flux measurements are required, a semiconductor detector with a current-measuring voltage-to-frequency converter provides a simple system with excelient linesrity and stability.

\section{DETECTOR CHARACTERISTICS AND FABRICATION TECHNIQUES}

For the following discussion on detector properties, it is useful to recall a few basic features of semiconductor detectors. Figure 2 shows a schematic cross section of a modern silicon lithium-drifted detector. The detector is basically a reverse biased $n^{+}-i-p^{+}$diode. When an incident photon interacts in the intrinsic region of the diode tracks of electron-hole pairs are produced. In the presence of an eleztiti: field these electron-hole pairs separate and rapidly drift to the detector contacts. The charge collected, $Q_{i}$, is proportional to the energy, $\mathrm{E}$, of the incident radiation:

$$
\mathbf{Q}_{\mathbf{q}}=\mathrm{Eq} / \mathrm{c}
$$


where $q=1.6 \times 10^{19}$ coulombs,$e(S i)=3.6 \mathrm{eV}$ and $e(G e)=2.98 \mathrm{eV}$. The rms statistical fluctuation in $Q_{1}, \delta Q_{2}$ is:

$$
\delta Q_{3}=q(F E / e)^{1 / 2}
$$

where $F$ is the Fano fuctor, which desc:ibes the deviation from normal statistics. The best value currently accepted for $F$ is $\approx 0.1$ for both silicon and gs:manium. Values much greater than this are indicative of charge-collection problems in the material. The full width half maximum energy resolution, $\delta \mathrm{E}_{f w H M}$, of a detector system is $\mathrm{g}$ vèn by:

$$
\delta \mathrm{E}_{\mathrm{FWHM}}=2.35 \mathrm{e}\left(\delta \mathrm{Q}^{2}+\delta \mathrm{Q}_{\mathrm{e}}^{2}\right)^{1 / 2} / \mathrm{q}
$$

where $\delta Q_{e}$ is the noise from the electronic amplifier.

An ideal radiation detector has the following set of requirements: lected.

a) The sensitive region must not haye trapping centers, which would affect the amount of charge co!-

b) The contacts must be thin enough to allow passage of the radiation without appreciable intensity or energy loss. $\mathrm{V} / \mathrm{cm}$.

c) The contacts need to remain non-injecting for reverse-bias field strengths of $\mathbf{E}=10^{2}$ to $10^{4}$

d) The detector surfaces have to be passivated against detrimental effects from the environment.

e) The noise caused by reverse leakage must be small compared to signal fluctuations.

Thin silicon detectors (less than $0.5 \mathrm{~mm}$ thick) are generally used as charged-particle detectors, have thin contacts on both sides and are operated with the total volume active (totally depleted). The three basic technologies of fabrication are surface barrier, diffused junction and ion implantation. Details of these procedures have been presented elsewhere and will not be repeated here, since they are not usually used as photon detectors.

Thick silicon detectors are fabricated by lithium ion compensation of $\mathrm{p}^{+}$type single crystals. The basic principle of this process is that lithium is a very mobile ion, which will drift interstitially under the influence of an applied electric field and which will compensate the nascent boron impurities to produce a nearly intrinsic region. The fabrication process begins with a lithium diffusion into the p-type silicon. After a $\mathrm{p}^{+}$contact is formed by gold evaporation on the opposite side and a surface treatment has been done to minimize the leakage current, the device is put on to "drift". The "drift" consists of applying a reverse bias ( $500-1000$ volts) and heat (11C- $350 \mathrm{deg} \mathrm{C}$ ) to the device. It typically takes about 4 weeks to compensate a $5 \mathrm{~mm}$ thick device. Silicon detectors thicker than $6 \mathrm{~mm}$ are not usually prepared because of the very long drifting times which are necessary. After the drifting is complete, the thick lithium diffused layer is sometimes lapped of and a thinner contact made. The edge protection of these devices is frequently accomplished by coating the surfaces with paints, varnishes and other materials which do not substantially alter the surface states. A more integral surface trealment using amorphous silicon has re' sntly been developed by Walton et al [3]. The long term stability of these detectors is determined by the quality of the surfacc passivation. As a result the coatings applied by various manufacturers are normally proprietary.

Germanium detectors were formerly fabricated by lithium ion compensation in much the same manner as silicon detectors. Today commercial manufacturers make germanium detectors from starting 
material which is pure enough so that no compensation is necessary. Although it is difficult to refine germanium to the required purity leveis, the advantages of using high purity material are substantial. These advantages include improved long-term stability, a wider choice of contact techniques, the possibility of fabricating multi-element detectors, and the ability to anneal radiation-damaged detectors in situ. Techniques have been developed which use SiO, various chemicals or amorphous $G$ e to passivate the exposed $\mathrm{Ge}$ detector surface[4]. Several manufactures now make portable germanium detectors for which it is necessary to cool the detector only when in use. A wide variety of detector geometries are now commercially available with active volumes up to $200 \mathrm{~cm}^{3}$.

\section{ELECTRONIC SYSTEMS FOR DETECTORS}

The electronic systems associated with these detectors are important components of the detector system, especially at low energies $(<10 \mathrm{kcV})$, where the energy resolution is dominated by electronic noise rather than by the detector. The first stage of the preamplifier for these systems is often mounted within the cryostat with very short leads from the detector to the first FET amplifier stage. The FET's used in this stage are usually matched in capacitance to the detector and are. often removed from their package and remounted in a low-loss package. Resistor, pulsed-optical and transistor reset feedback systems are all used to restore the charge on the seedback capacitor used to make the preamp chargesensitive. Resistor feedback systems have the disadvantage that the feedback resistor has different properties at different frequencies which makes the pole-zero compensation network difficult to optimize. Optical feedback systems are used in very low noise systems. The disadvantage of them is that the LED-FET preamp package is very difficult to fabricate. Although the transistor reset preamp has slightly higher noise than the pulsed optical systems, it has the advantages that the preamp package is easier to fabricate and in multi-detector systems the interchannel crosstalk is much less. Figure 3 is a pholograph showing the integrated detector/FET moduie which is used at the Lawrence Berkeley Laboratory (LBL) for multidetector systems. This preamplifier has the feature that a ground point obtained from the vicinity of the signal output driver is brought out and used in the amplifier to make the system less sensitive to electrical noise. The charge sensitive loop is designed to provide a fast risetime ( $20 \mathrm{~ns}$ ), so that signal integration in the fast processing part of the amplifier can be determined by an integrator in the amplifier. Test signals can also be injected through the detector capacitance to provide a reference pulse of high accuracy $(0.1 \%)$ and low noise.

Much of the literature dealing with optimizing pulse shape to obtain the best energy resolution with these detectors focuses on cases where parallel (step) and series(delta) noise are present and where the choice of the amplifier time constant can be made to equalize these two terms. With presently available FETs and using small-area detectors, the optimum shaping time may well be in the $100 \mu \mathrm{s}$ range. In applications where high counting rates are essential, such as are common with synchrotron $x$-ray sources, pulse shaping times in this range are not acceptable and shorter times must be used. Under these circumstances the dominant noise source is series (delta) noise caused primarily by fluctuations in the channel current of the FET.

The problem of optimizing pulse shape is therefore simplified to that of choosing a pulse shape that, for a given total duration, gives tbe best signal-to-noise ratio when only series noise is present. In this case the symmetrical triangular pulse shape is best but derivation of such a pulse processor has presented designers with difficulties, since the use of delay lines produces problems of gain sensitivity to temperature variations. Recently Gnulding, Landis and Madden [2] have developed an amplifier which uses a weighted mixing of the outputs of gain stages which are already present in most Gaussian shaping amplifiers. Waveforms from the cutputs of the first, second and third integration stages are mixed in the ratio of 0.324:0.168:1.000 respectively to pioduce the approximately symmetric triangular autput pulse. Figure 4 shows the calculated shape of the three weighted waveforms, the resulting triangular pulse and a true symmetrical triangular pulse. The output pulse differs from the true symmetrical triangular pulse in that the peak and tail of the pulse are rounded. This rounding does not decrease the energy resolution but does 
increase the pulse pile-up effects slightly. The series noise of this amplifier is $8 \%$ less the: that of many modern spectroscopy amplifiers which use quasi-Gaussian waveforms.

An important part of all molern spectrometer amplifiers is a method to prevent the analysis of pulses whose amplitude is subjee: to interference from other signals in close time proximity. This is generally achieved by a "pile-up rejector", which consisis of a gate at the output of the main amplifier channel and a parallel fast-inspection channel where sigrals are differentiated to form narrow pulses from which a fast discriminator produces logic signals. These logic signals are examined by a pile-up rejector, which, by measuring time intervals between signals, senses when pile-up effects in the main amplifier may distort the output signal amplitude. When there is no pile-up a data valid logic signal allows the production of the output pulse. If there is pile-up no sigial is produced and the signal gate inhibits the output pulse.

In general a short, well determined resolving time is desired in the fast inspection channel to minimize the acceptance of pulses which occur within this time and result in higher general background and "sum" peaks in the output spectrum. Unfortunately most modern spectrometer amplifiers do not provide a very well determined resolving time since they use integration and single delay line or simple RC pulse shaping. As shown in Fig. 5(a), the long exponential tail on the back edge of the pulse causes the resolving time to be very dependent on the pulse amplitude and therefore very difficult to determine in cases with a wide range of pulse amplitudes. In the amplifier developed by Goulding, Landis and Madden this problem was solved. They used a known RC integrator combined with an asymmetrical bipolar delay line pulse shaper to produce an output pulse that returns to the baseline at a time equal to the total bipolar pulse width as shown in Fig. 5(b). This pulse shaping also is a reasonable approximation to a symmetrical inangular shape which gives the best signal-to-noise ratio when series noise is dominant.

To achieve maximum throughput, the normal pile-up inspection system was modified to accept pulses in which, although there is pulse overlap, the pulses do not overlap when they are at their peak amplitude and therefore can be measured correctly. The events which are accepted by this system and normally rejected by a conventional system are shown in cise 2 of Fig 6 . Normal pulse pile-up rejectors reject the second pulse if the pulse has not returned to near the baseline before the second begins and therefore the second pulse in both case 1 and case 2 pulses are rejected. The effective dead time for loss calculations is equal to $T_{d l}$ where:

$$
T_{\mathrm{dl}}=2 T_{1}+T_{2}
$$

where $T_{1}$ is the time from the start of the pulse to maximum amplitude and $T_{2}$ is the time from maximum amplitude to the end of the pulse. The improved pile-up inspector still rejects pulses which overlap at the pulse peak (case 1) but accepts pulses where the overlap is before the pulse peak. This system imposes an effective dead time, $T_{d 2}$, given by:

$$
T_{\mathrm{d} 2}=T_{1}+T_{2}=T_{w}
$$

where $T_{w}$ is the total pulse width. For perfect triangular pulse shaping, where $T_{1}=T_{2}, T_{d j}=1.5 T_{d 2}$, which is a 50\% improvement in throughput. For the new $\mathrm{LBL}$ system with $T_{\mathrm{dl}}=4 \mu \mathrm{s}$ and $T_{\mathrm{d} 2}=5 \mu \mathrm{s}$, the dead time is $9 \mu \mathrm{s}$, while with a standard system the dead time is $13 \mu \mathrm{s}$. The improvement in peak output rate is therefore about 35\%. Figure 7 shows the output counting rate as a function of the input rate for the two systems. As can be seen in the figure, the peak output rate is improved by about $35 \%$ by using the improved pile-up rejector. In high rate experiments, such as are common at synchrotron sources, this improvement will be very useful.

Semiconductor detectors are also useful in experiments which require a high-flux detector with excellent linearity and stability. In this application, the detector current can be measured with a simple integrated-circuit amplifier followed by a voltage-to-frequency converter. Flux information has been 
obtained from a fan beam in small segments using a multi-element detector with separate readout chanrels on each element [5]. With modern fabrication techniques, complex detector patterns are possible. LBL is currently fabricating a silicon lithium-drifted detector which will have an active volume $150 \mathrm{~mm}$ long, 3 $\mathrm{mm}$ wide and $5 \mathrm{~mm}$ thick, consisting of a linear array of 300 elements. Each element will be $0.4 \mathrm{~mm}$ by 3 $\mathrm{mm}$ and have a center-to-center spacing of $0.5 \mathrm{~mm}$. The detector will be used with 300 separate channels of readout electronics in an experiment evaluating the application of synchrotron radiation for digital angiography [6].

\section{PROPERTIES OF DETECTOR SYSTEMS}

An important consideration in multi-element detector systems is the crosstalk between detector elements. Electronic crosstalk must be minimized by carefuily shielding both the leads from each elcment and the preamplifiers. There is also interaction between detector elements when photons produce charge between detector elements. The electron-hole pairs are be distributed between elements and give a distorted energy spectrum in both channels. This sharing of charge was measured at the Stanford Synchrotron Radiation Laboratory using a $33 \mathrm{keV}$ photon beam collimated to $0.1 \mathrm{~mm}$ diameter and a multielement lithium-drifted $5 \mathrm{~mm}$ thick silicon detector with 60 elcments in a linear array. Each element had a width of $0.4 \mathrm{~mm}$ with a spacing between elements of $0.1 \mathrm{~mm}$. The detector was cooled to $-30 \mathrm{deg} \mathrm{C}$. Each of two adjacent elements gave an energy resolution of $4.5 \mathrm{keV}$ when connected to room-temperature charge-sensitive preamplifiers. The energy spectrum was measured in both channels as the beam was scanned in $0.02 \mathrm{~mm}$ steps across the region between the elements. As the beam was moved from within the element and towards the adjacent element, the full-energy peak intensity decreased and low-energy spectrum increased as shown in Fig. 8. In Fig. 9 the total energy collected from 7 to $45 \mathrm{keV}$ and the fullenergy peak intensity ( 29 to $37 \mathrm{keV}$ ) measured in each channel as a function of beam position is given. When these detectors are used in a current readout mode the charge is shared smosthly between the elements. However, when these detectors are used for individual pulse counting, it may be useful to utilize these stared charge events by detecting coincident events with fast logic and summing their energies. In other experiments it may be better to reduce the spectral background by rejecting these events.

The time resolution of semiconductor detectors at photon energies below $50 \mathrm{keV}$ is limited by electronic noise and not by the variable pulse shape from pulses interacting in different segions of the detector. A planar semiconductor detector can give a time resolution of better than $20 \mathrm{nsec}$ with $5 \mathrm{keV}$ photons when a constant-fraction discriminator is used.

\section{APPLICATIONS}

Some of the applications in which these systems have been used with synchrotron sources are trace element analysis, fluorescence EXAFS, energy-dispers.ve powder diff:sction, digital subtraction angiography, and anomalous $\mathrm{x}$-ray scattering near absorption edges.

1. Trace element analysis experiments using $x$-ray fluorescence with synchrot ron sources has been done using semiconductor detectors by a number of groups including Sparks [7] and Gilfrich [8]. Sparks has shown theoretically that semiconductor detectors could be used to measure elemental concentrations down to several $\mathrm{ppb}$ [9]. The development of $\mathrm{x}$-ray beams with diameters of a few $\mu \mathrm{m}$ will soon make sensitive $x$-ray microprobes possible. Semiconductor detectors could be used with these beams to give simultaneous imaging capability for many elements. The use of multi-element detector systems and highperformance pulse processors could make synchrotron sources even more useful in measuring dilute concentrations of elements in small samples.

2. In flucrescence EXAFS experiments, the output counting rate in the fluorescence peak is measured as a function of the incident photon energy. In these experiments it is therefore very important 
that the output counting rate change linearly with the input rate. Semiconductor detectors are used in these experiments only when the element to be studied is present in only low concentration. For example, Klein et al have used these detectors in EXAFS studies of biological systenss [10,11]. The pile-up rejector described in this paper would improve the counting rate for such detector systems. For experiments on very dilute systems, a multi-element detector could also be used to increase the total counting rate.

3. Energy-dispersive $x$-ray powder diffraction experiments require good energy resolution over a wide energy range to accurately measure d spacings of crystals. For example, Skelton et al have used this method with a semiconductor jetector to study a variety of samples $[12,13]$. With synchrotron radiation sources, the available input count rate of these spectrometers can be greater than $100 \mathrm{kHz}$. As with trace element analysis, higher counting rates may be obtained with improved detector electronics.

4. Digital-subtraction angiography with synchrotron radiation requires a one-dimensional detector with many elements to measure the flux transmitted through a sample as a function of position. The silicon detector used for this technique illustrates how a silicon multi-element detector can be used with synchrotron radiation to measure high fluxes with excellent linearity and stability $[6,14]$.

5. Semiconductor detectors have also been used in in anomalous-scattering experiments $[15,16]$. In these experiments the detector was mounted on a goniometer and rotated as the experiment was done. The detector was used with a single-channel analyzer to count elastic-scattered scattered photons and to reject compton and fluorescence photons. Replacing a Nal detector with a germanium detector significantly improved the signal-to-noise ratio and the overall stability.

Other applications of these detectors will certainly be found as synchrotron sources become more available and the intensity of the $x$-ray beams is improved.

\section{CONCLUSIONS}

Semiconductor spectrometer systems provide very useful detector systems for experiments at synchrotron facilities. Their excellent bandwidth, erergy resslution, linearity and count-rate parformance can be widely applied with the high-irtensity sources that synchrotrons provide. Silicon detectors are valuable printarily in the photon range below $20 \mathrm{keV}_{2}$ and germanium detectors in the range above $20 \mathrm{keV}$. The development of a transistor-reset preamplifier and a triangular pulse-shaping amplifier has improved the energy resolution at shorter amplifier time constants by $8 \%$ and increased the pulse throughput by $35 \%$. There are a wide variety of experiments which already use seciconductor spectrometers. The demanding requirements of experiments with synchrotron sources will result in increasing use of semiconductor detectors at these facilities.

\section{ACKNOWLEDGEMENTS}

The information provided by D. Landis, N. Madden, M. Maier and P. Luke is appreciated. This work was supported by the Director's Office of Eneigy Research, Office of Health and Environmental Research, U.S. Department of Energy under Contract No. DE-AC03-76SF00098. 


\section{REFERENCES}

1. D.A. Landis, C.P. Cork, N.W. Madden asd F.S. Goulding, IEEE Trans. Nucl. Sci., 29 (1982) 619.

2. F.S. Goulding, D.A. Landis and N.W. Madden, Lawrence Berkeley Laboratory report LBL-14464, (1983).

3. J.T, Walton, R.H. Pehl, Y.K. Wong and C.P. Cork, to be published in IEEE Trans. Nucl. Sci. Conference on Nuclear Jcience, San Francisco, 1983.

4. W.L. Hansen. E.E. Haller and G.S. Hubbard. IEEE Trans. Nucl. Sci., 27 (1980) 247.

5. H.D. Zeman, E.B. Hughes, L.E. Campbell, R. Hofstader, R.L. Kith, T.J. Krolicki, J. Rolfe, J.P. Stone,

S. Wilson, E Rubenstein, A.C. Thompson and J.T. Walton, IEEE Trans. Nucl. Sci., NS-29,(1982) 442.

6. E.B. Hughes, H.D. Zeman, L.E. Campbell, R. Hoistader, U. Meyer-Berkout, J.N. Otis, J. Rolfe, J.P. Stone, S. Wilson, E. Rubensteis, D.C. Harrison, R.S. Kernof, A.C. Thompson and G.S. Brown, Nucl. Instr. and Meth., 208 (1983) 665.

7. C.J. Sparks, S. Raman, E Ricci, R.V. Gentry and M.O. Krause, Phys. Rev. Lett., 40 (1978) 507.

8. J.V. Gilfrich, E.F. Skelton, D.J. Nagel, A.W. Webb, S.B. Quadri and J.P. Kirkland, Adv. in X-Ray Analysis, 26 (1983) 313.

9. C.J. Sparks, in "Synchrotron Radiation Researıh", H. Winick and S. Doniach, eds., Plenum Publishing Co., New York (1980).

10. J. Kirby, A Robertson, J. Smitb, S. Copper and M. Klein, JACS, 103 (1981) 5529.

11. J. Kirby, D. Goodin, T. Wydrzynsīi, A. Robertson and M. Klein, JACS, 103 (1981) 5537.

12. E.F. Skelton, Adv. in Earth and Planetary Sci., 12 (1982) 61.

13. E. F. Skelton, J. Kirkland and S. Qadri, J. Appl. Cryst., 15 (1982) 82.

14. A.C. Thompson, F.S. Goulding, H.A. Sommer, J.T. Walton, E.B. Hughes, J. Rolfe and H.D. Zeman, IEEE Trans. Nucl. Sci., NS-29 (1982) 793.

15. W. Warburton, private communication.

16. S. Aur, D. Kofalt, Y. Waseda, T. Egami, H.S. Chen, B. Teo and R, Wang, University of Pennsylvania preprint. 


\section{FIGURE CAPTIONS}

1. Efficiency of silicon and germanium detectors as a function of energy. The low energy efficiency with a thin silicon dead layer, with a $\mu \mathrm{m} \mathrm{Be}$ window and with $2 \mathrm{~cm}$ of air between the source and the detector are also shown. The fluorescent $\mathrm{K} \alpha$ photon energies of some elements are indicated above the efficiency curves.

2. Schematic cross section of a modern silicon lithium-drifted detector.

3. Photograph of an integrated detector/FET preamplifier module which is used in spectrometer systems at the Lawrence Berkeley Laboratory.

4. Calculated shape of the three weighted waveforms, and the resulting waveform from the new LBL amplifier. A true symmetrical triangular pulse is also shown for comparison.

5. Fast channel pulse shapes in (a) a conventional system and (b) in the new system. The widening of the resolving time for the conventional system as the pulse amplitude increases is shown.

6. Illustration of the various pile-up conditions. In the new pile-up rejector system events of case 2 are accepted whereas in normal pile-up rejectors they are rejected.

7. A plot of the output versus input counting rate for a system with the conventional pile-up rejector and with the new system.

8. Energy spectra for one element of a multi-element detector at different positions the detector contact edge using a $0.1 \mathrm{~mm}$ diameter, $33 \mathrm{keV}$ photon beam.

9. Total energy collected from 7 to $45 \mathrm{keV}$ (solid lines) and the full-energy peak intensity (dotted lines) from two adjacent detector elements as a function of the position of a 0.1 mnl diameter, $33 \mathrm{keV}$ photon beam. 


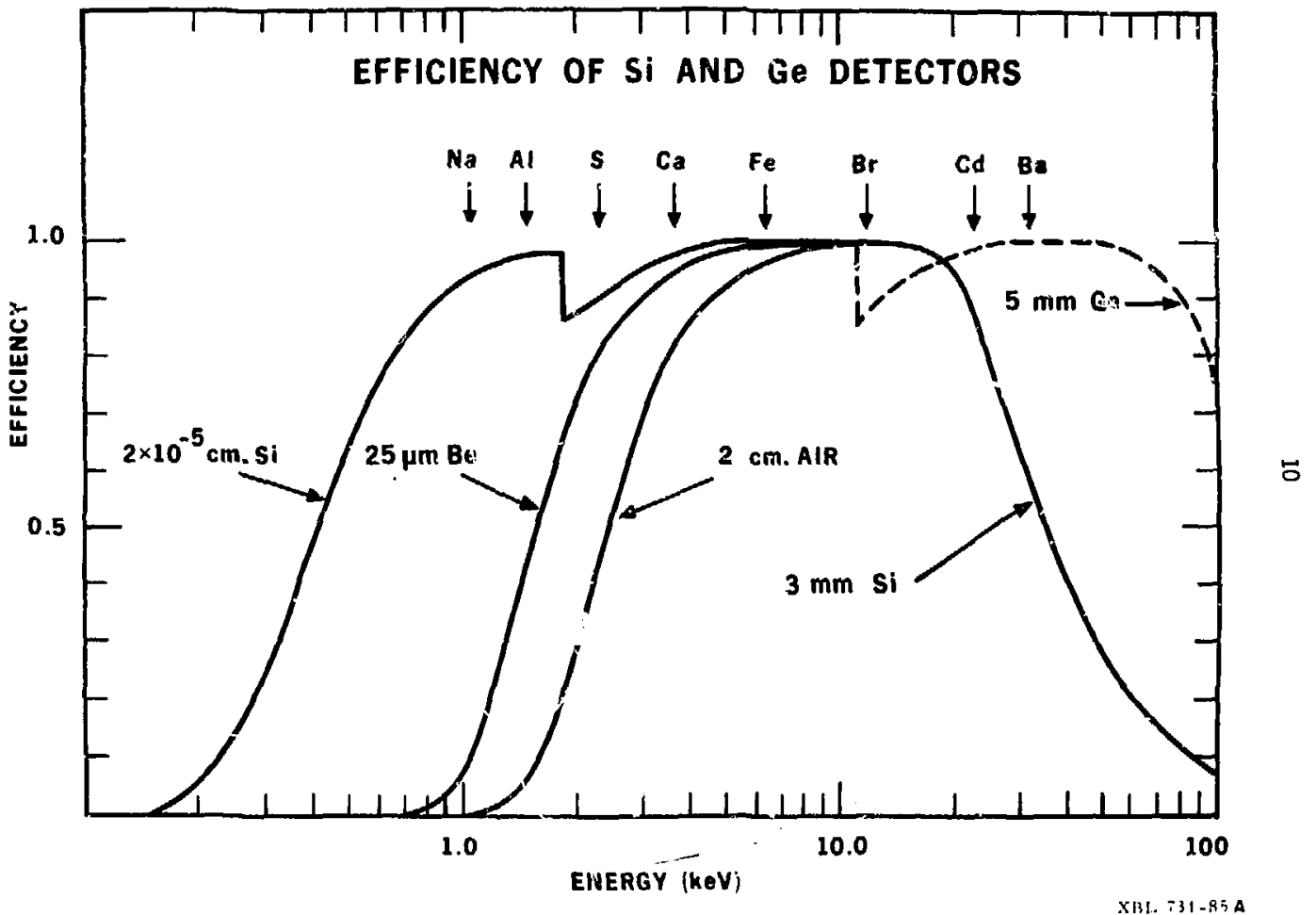

Fig. 1. Efficiency of silicon and gemianium detectors as a function of energy. The low energy efficiency with a thin silicon dead loyer, with a $\mu \mathrm{m}$ Be window and with $2 \mathrm{~cm}$ of air between the source and the detector are also shown. The fluorescent $k \alpha$ photon energies of some elements are indicated above the efficiency cumies. 


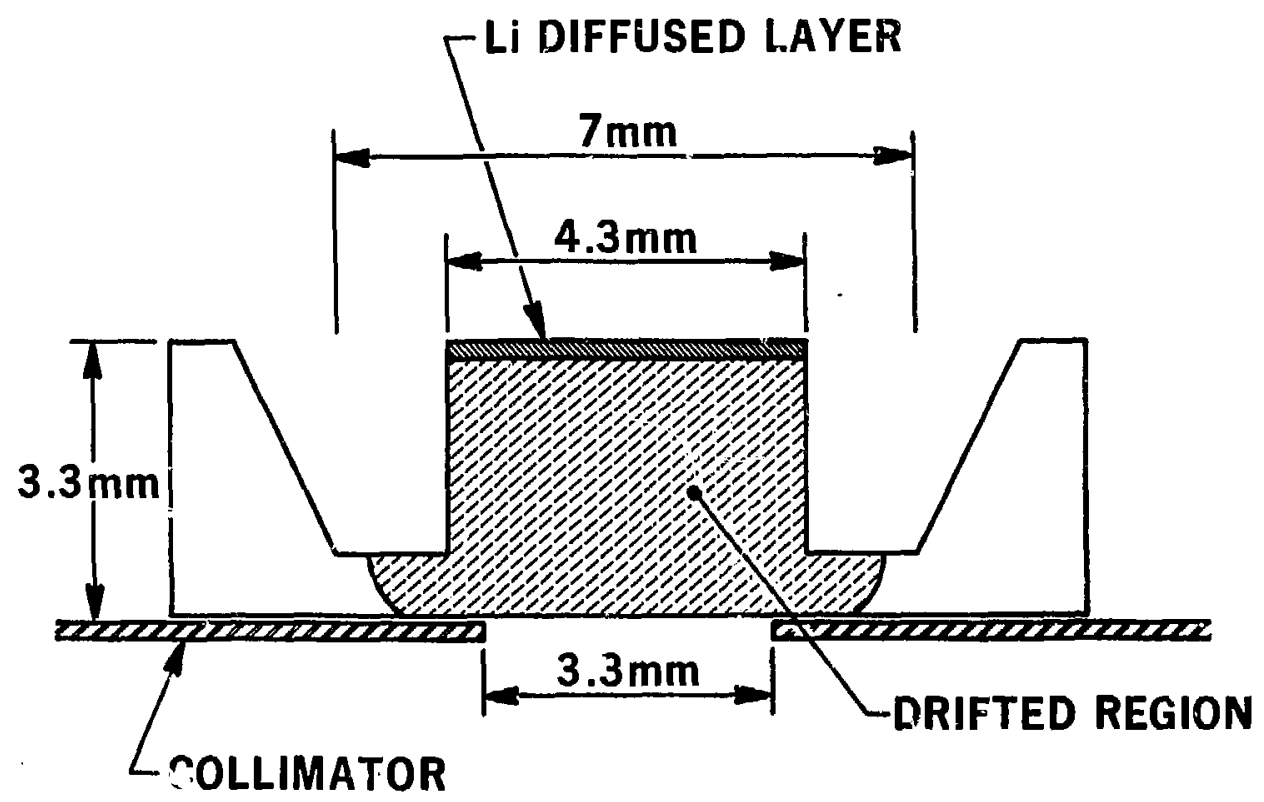

XBL $8210-2870$

Fig. 2. Schematic cross section of a modern silicon lithium-drifted detector. 


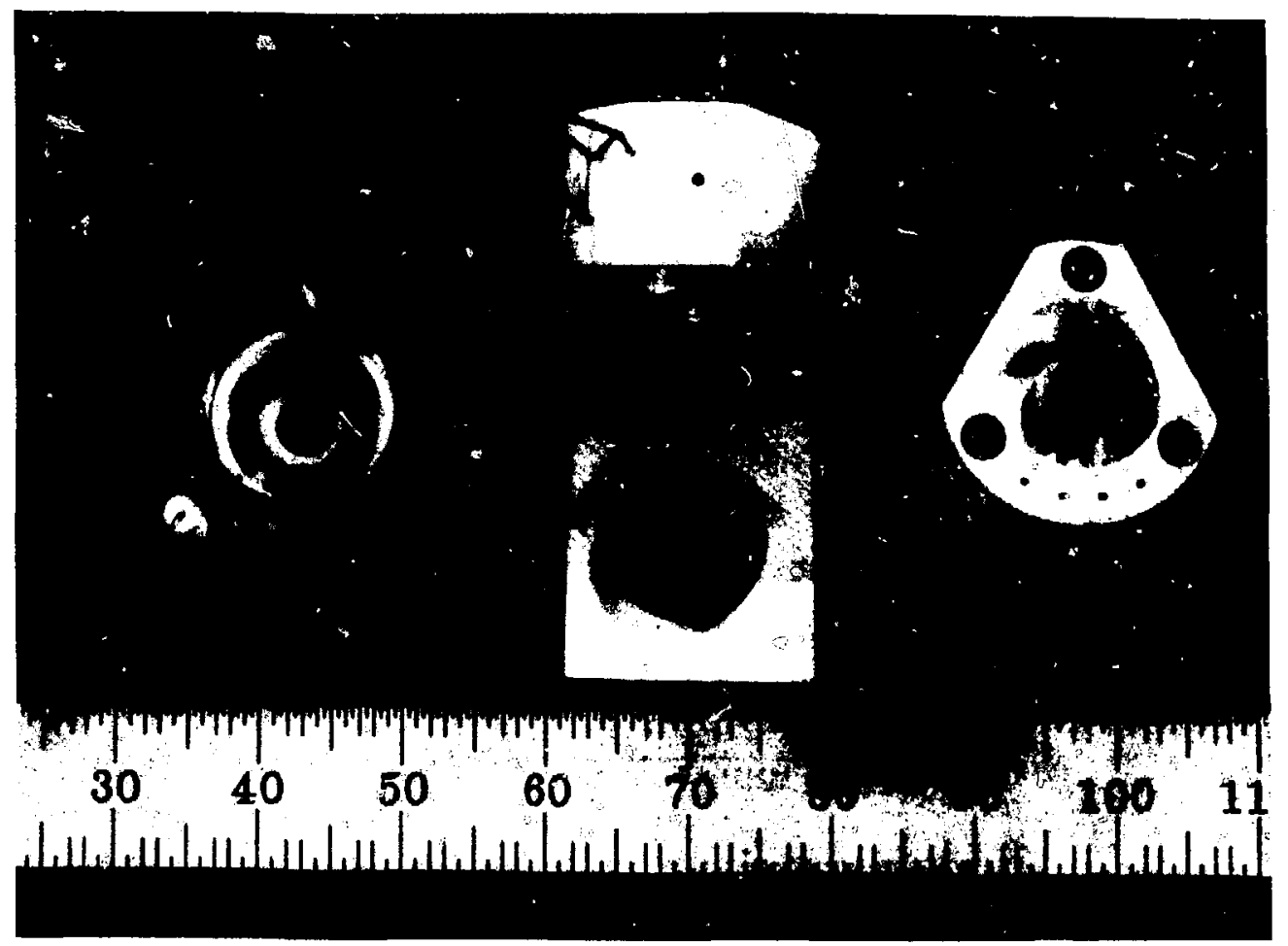

CBB 8210-8787

Fig. 3. Photograph of an integrated detector/FET preamplifier module which is used in spectrometer systems at LBL. 


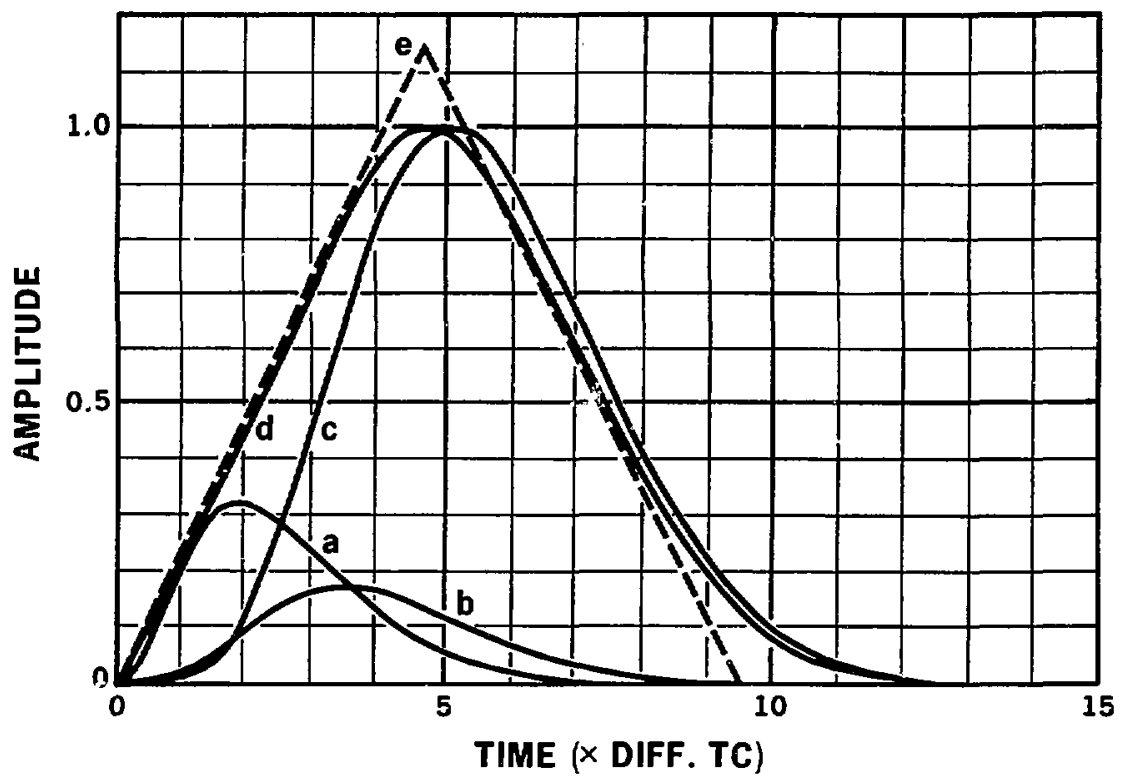

XBL $8210-2874$

Fig. 4. Calculated shape of the three weighted waveforms, and the resulting waveform from the new LBL amplifier. A true symmetrical triangular pulse is also shown for comparison. 

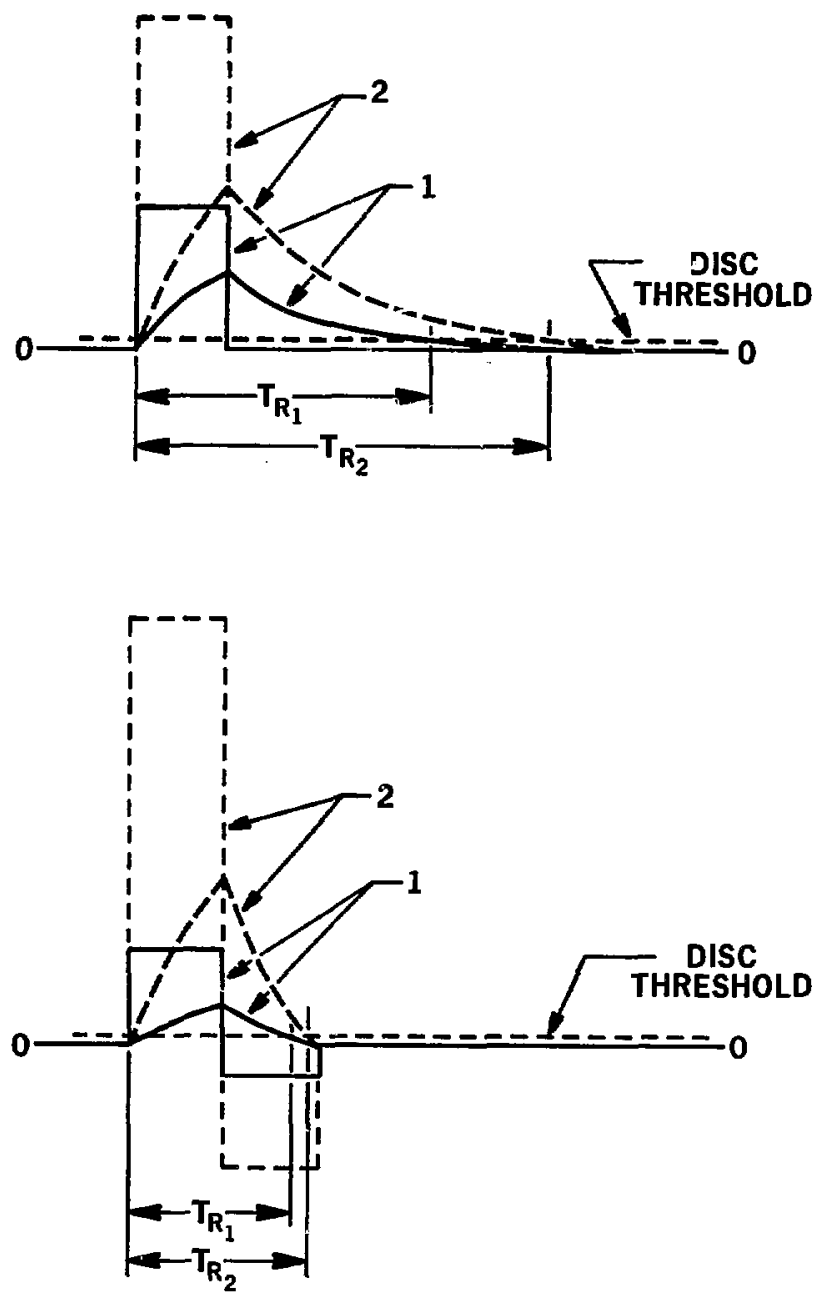

XBL $8210-2871$

Fig. 5. Fast channel pulse shapes in (a) a conventional system and (b) in the new system. The widening of the resolving time for the conventional system as the pulse amplitude increases is shown. 


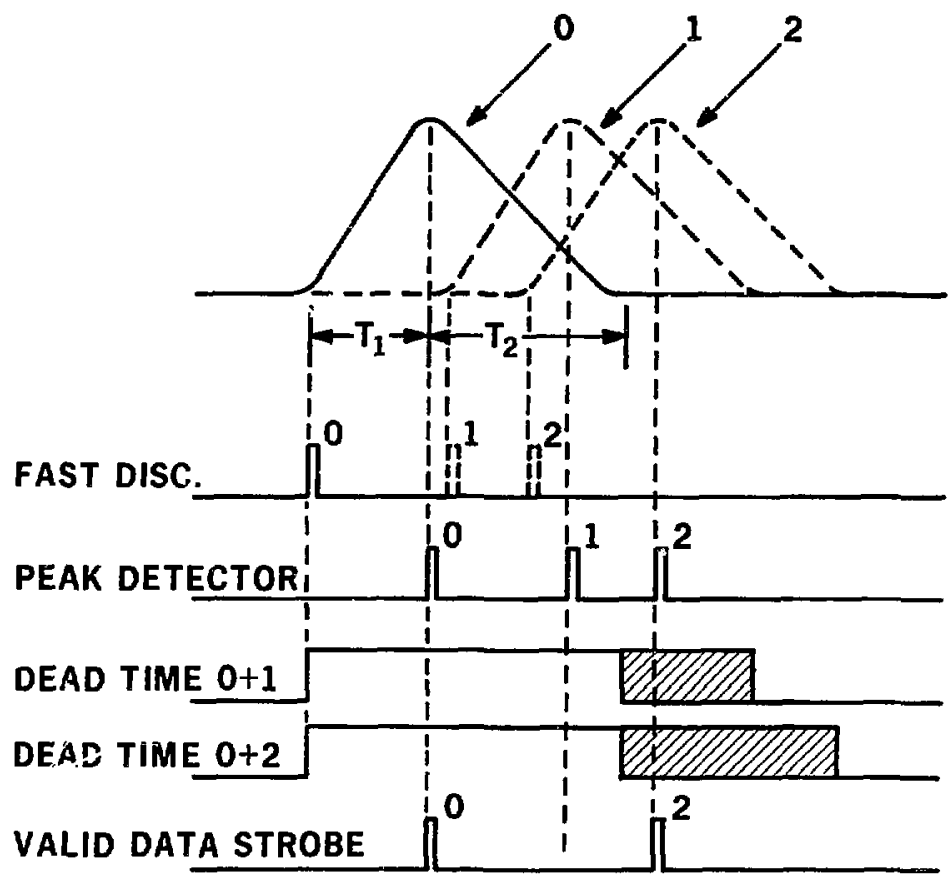

XBL $8310-12120$

Fig. 6. Illustration of the various pile-up conditions. In the new pile-up rejector system events of case 2 are accepted whereas in normal pile-up rejectors they are rejected. 


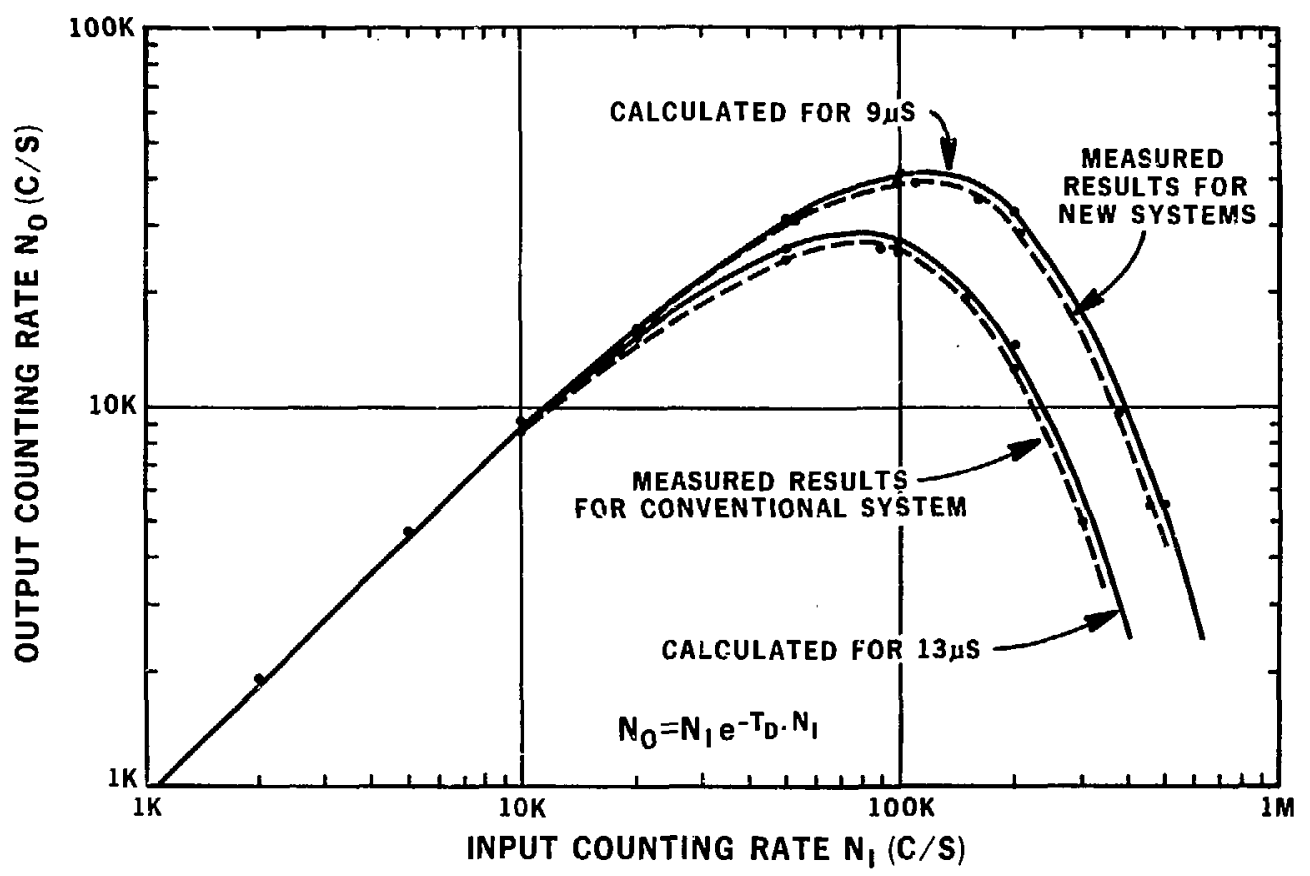

Fig. 7. A plot of the cutput versus input counting rate for a system with the conventional pile-up rejector and with the new system 


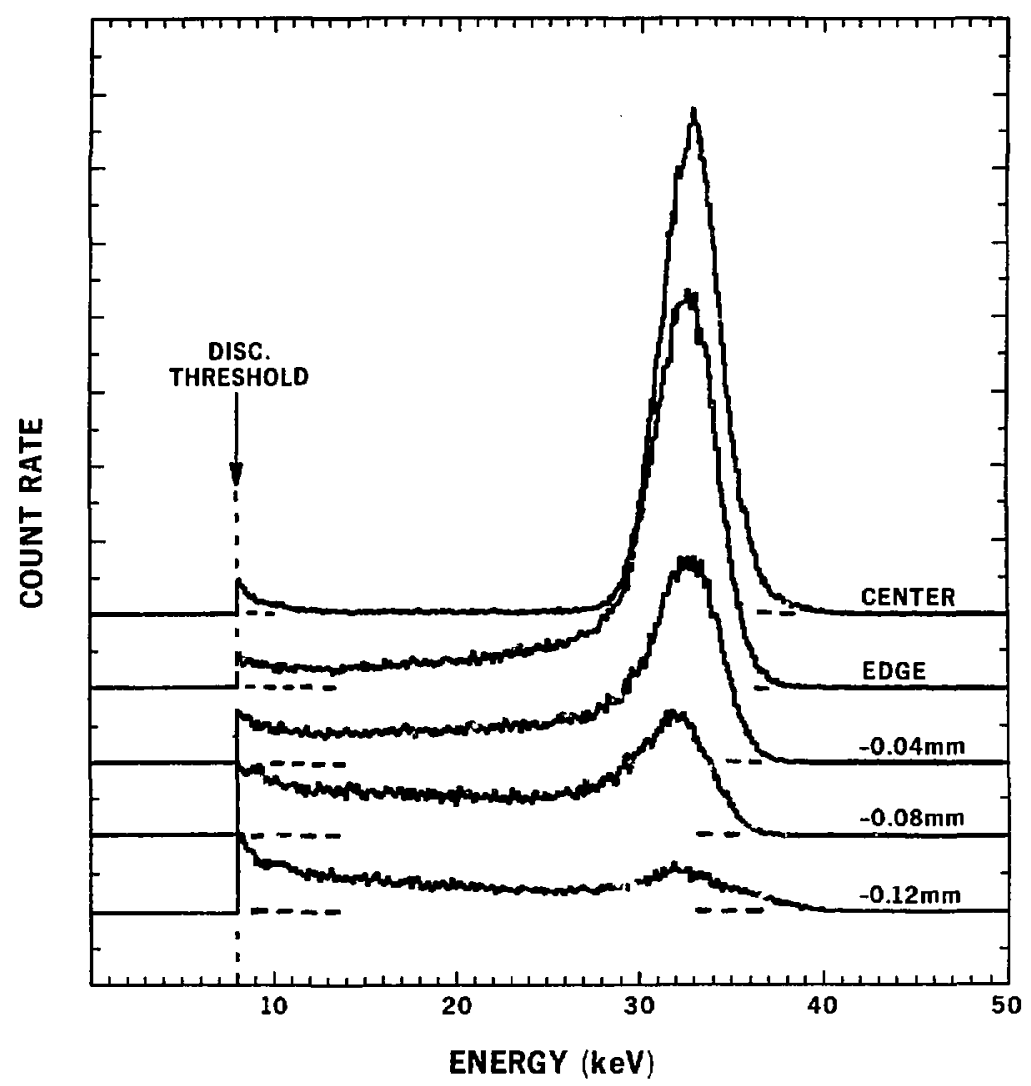

XBL 8310-11915

Fig. 8. Energy spectra for one element of a multi-element detector at cifferent positions on the detector contact edge using a $0.1 \mathrm{~mm}$ diameter, $33 \mathrm{keV}$ photon beam. 


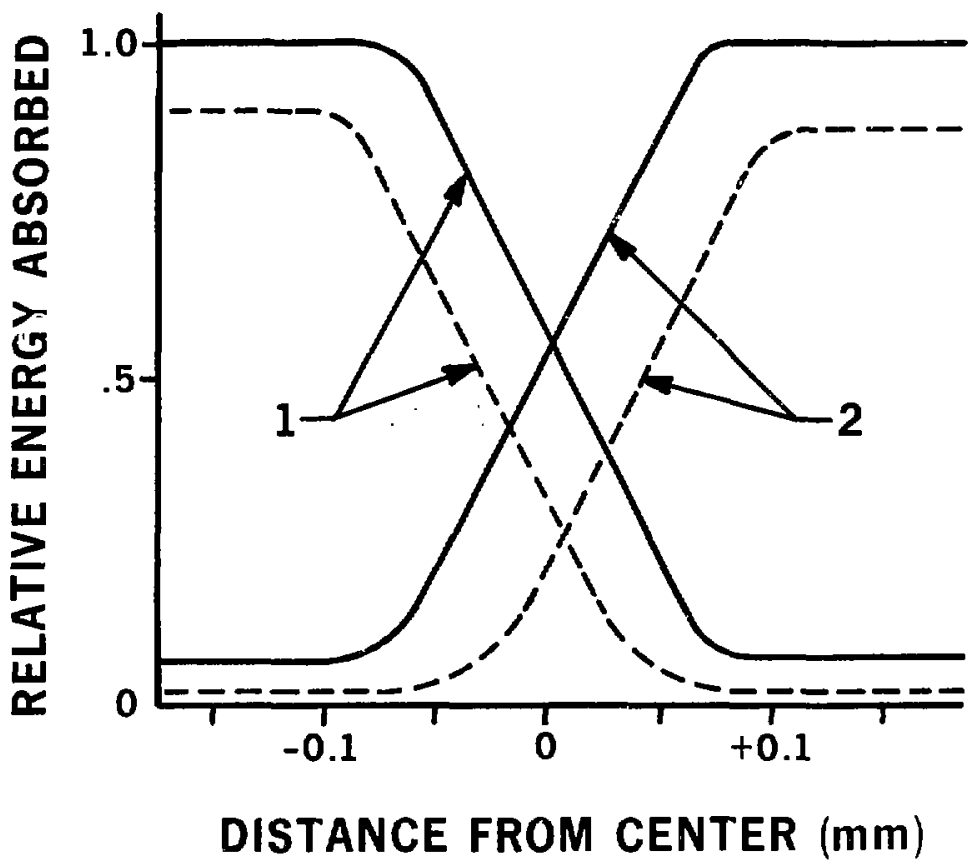

XBL 8310-12119

Fig. 9. Total energy collected from 7 to $45 \mathrm{keV}$ (solid lines) and the full-energy peak intensity (dotted lines) from two adjacent detector elements as a function of the position of a $0.1 \mathrm{~mm}$ diameter, $33 \mathrm{keV}$ photon beam. 
This report was done with suppor from the Department of Energy. Any conclusions or opinions expressed in this report represent salely those of the aulhor(s) and not necessarily those of The Regents of the University of Califernia, the Lawrenee Berkeley Laboratory or the Deparment of Energy.

Referenee to a company or product name does nol imply approval or recommendation of the product by the University of Caifornia or the U.S. Department of Energy to the exclusion of others that may be suitable. 